\title{
A Versatile Nanoplatform for Broad-spectrum Immunotherapy by Reversing Tumor Microenvironment
}

Ao Chen, ${ }^{\dagger, \#, \nabla}$ Fan Yang, ${ }^{\dagger, \nabla}$ Jing Kuang, ${ }^{\dagger}$ Yuan Xiong,${ }^{\S, \|}$ Bo-Bin Mi, ${ }^{\S, l}$ Ying Zhou, ${ }^{\dagger}$ Jing-

Jing Hu, ${ }^{\perp}$ Shu-Jun Song, ${ }^{\dagger}$ Tao Wan, ${ }^{\dagger}$ Zhong-Zhong Wan, ${ }^{\dagger}$ Hong-Yang Huang, ${ }^{\#}$ Xin-

Run Li, ${ }^{\dagger}$ Wen Song, ${ }^{*} \dagger$ and Wen-Xiu Qiu ${ }^{*}$,

${ }^{\dagger}$ Institute of Biology and Medicine, College of Life Science and Health, Wuhan

University of Science and Technology, Wuhan, Hubei 430081, P. R. China

‡Institute of Pathology, Tongji Hospital, Tongji Medical College, Huazhong

University of Science and Technology, 430030, Wuhan, P. R. China

${ }^{\S}$ Department of Orthopaedics, Union Hospital, Tongji Medical College, Huazhong

University of Science and Technology, Wuhan 430022, P. R. China

${ }^{\|}$Hubei Province Key Laboratory of Oral and Maxillofacial Development and

Regeneration, Wuhan 430022, P. R. China

${ }^{\perp}$ State Key Laboratory of Biogeology and Environmental Geology, Faculty of

Materials Science and Chemistry, China University of Geosciences, Wuhan 430074,

P. R. China

\#Department of Pathology, Li Ka Shing Faculty of Medicine, The University of Hong Kong, Hong Kong 999077, P. R. China

\section{Corresponding Authors}


E-mail: songwen@wust.edu.cn (W. Song),

E-mail: wx_qiu1816@163.com (W. Qiu) 


\section{Contents}

Characterization of chimeric peptides (Figures S1-S3)

DSL analysis of CP (Figures S4)

H\&E staining of main organs (Figures S5)

Immunofluorescence observation of CRT exposure on and HMGB1 release in the 4T1

(Figure S6) and MCF-7 cells (Figures S7)

Western blotting of M1 and M2 population after different treatments (Figures S8) 


\section{Experimental Section}

Synthesis of copper peroxide: $0.5 \mathrm{~g}$ of polyvinylpyrrolidone (PVP, Mw 8000) was added to an aqueous solution of $\mathrm{CuCl}_{2}(5 \mathrm{~mL}, 0.01 \mathrm{M})$, and stirred at room temperature to dissolve. Then the solution of $\mathrm{NaOH}(5 \mathrm{~mL} 0.02 \mathrm{M})$ and $100 \mu \mathrm{L} \mathrm{H}_{2} \mathrm{O}_{2}$ were added to the mixed solution. The mixture was stirred at room temperature for $30 \mathrm{~min}$, centrifuged and washed with water to obtain copper peroxide nanoparticles.

Synthesis of Chimeric Peptide $C_{16}-P p I X$ : Chimeric peptide $\mathrm{C}_{16}-\mathrm{PpIX}$ was synthesized using standard Solid phase peptide synthesis (SPPS) using 2-chlorotrityl chloride resin. Briefly, the resin was soaked in anhydrous DMF for $30 \mathrm{~min}$, and then 2 equiv. (relative to the loading content of resins) of Fmoc-Arg(pbf)-OH and 6 equiv. of DIEA were added and reacted in DMF for $3 \mathrm{~h}$. Unreacted solution was removed by using anhydrous DMF three times. Unreacted reactive sites of resins were capped by 30 min reaction with a mixture of $\mathrm{CH}_{2} \mathrm{OH}$, DIEA and DMF (v/v/v=1.5:2:6.5). The resins were treated with $20 \%$ piperidine in DMF to remove the Fmoc protecting group. The next Fmoc-protected amino acid Fmoc-Arg(pbf)-OH was added to react with resins with 4.8 equiv. $\mathrm{HOBt}, 4.8$ equiv. HBTU and 8 equiv. DIEA for 2 h. Then $\operatorname{Arg}(\mathrm{pbf})$, $\operatorname{Arg}(\mathrm{pbf})$, Lys(Dde), and PA (C16) were conjugated to the resins by the same method. PpIX was conjugated to the resins after removing the Dde protected group by $2 \%$ hydrazine hydrate in DMF. Peptide was cleaved from resins by the solution of TFA, $\mathrm{H}_{2} \mathrm{O}$ and TIS with the ratio of $0.95: 0.25: 0.25$ for $2 \mathrm{~h}$. Finally, the filtrate was concentrated and the pure peptide was precipitated in cold ether. 
Synthesis of CP@mRBC-PpIX: The blood of healthy C57BL/6 mice was obtained from the eyeball, and the red blood cells were separated by centrifugation $(3000 \mathrm{rpm}$, $5 \mathrm{~min}$ ). The obtained red blood cells were added to the $0.25 \mathrm{mM}$ EDTA solution for lysis. The solution was centrifuged at 14,000 $\mathrm{g}$ for $30 \mathrm{~min}$ to obtain red blood cell membranes. Repeat the above steps until the color of the red blood cell membrane fades to obtain the erythrocyte membrane fragments. Then, the membrane fragments were lyophilized and stored at $-80^{\circ} \mathrm{C}$. The obtained erythrocyte membrane and the chimeric peptide were added to the PBS solution and stirred at room temperature for $24 \mathrm{~h}$, then copper peroxide $(\mathrm{m} / \mathrm{m}=1: 1)$ nanoparticles were added to the solution under ultrasonic bath for $1 \mathrm{~h}$. At the end, physically extruded 10 times by an Avanti mini extruder through a porous polycarbonate membrane $(400 \mathrm{~nm})$ to finally obtain $\mathrm{CP} @ \mathrm{mRBC}-\mathrm{PpIX}$. 

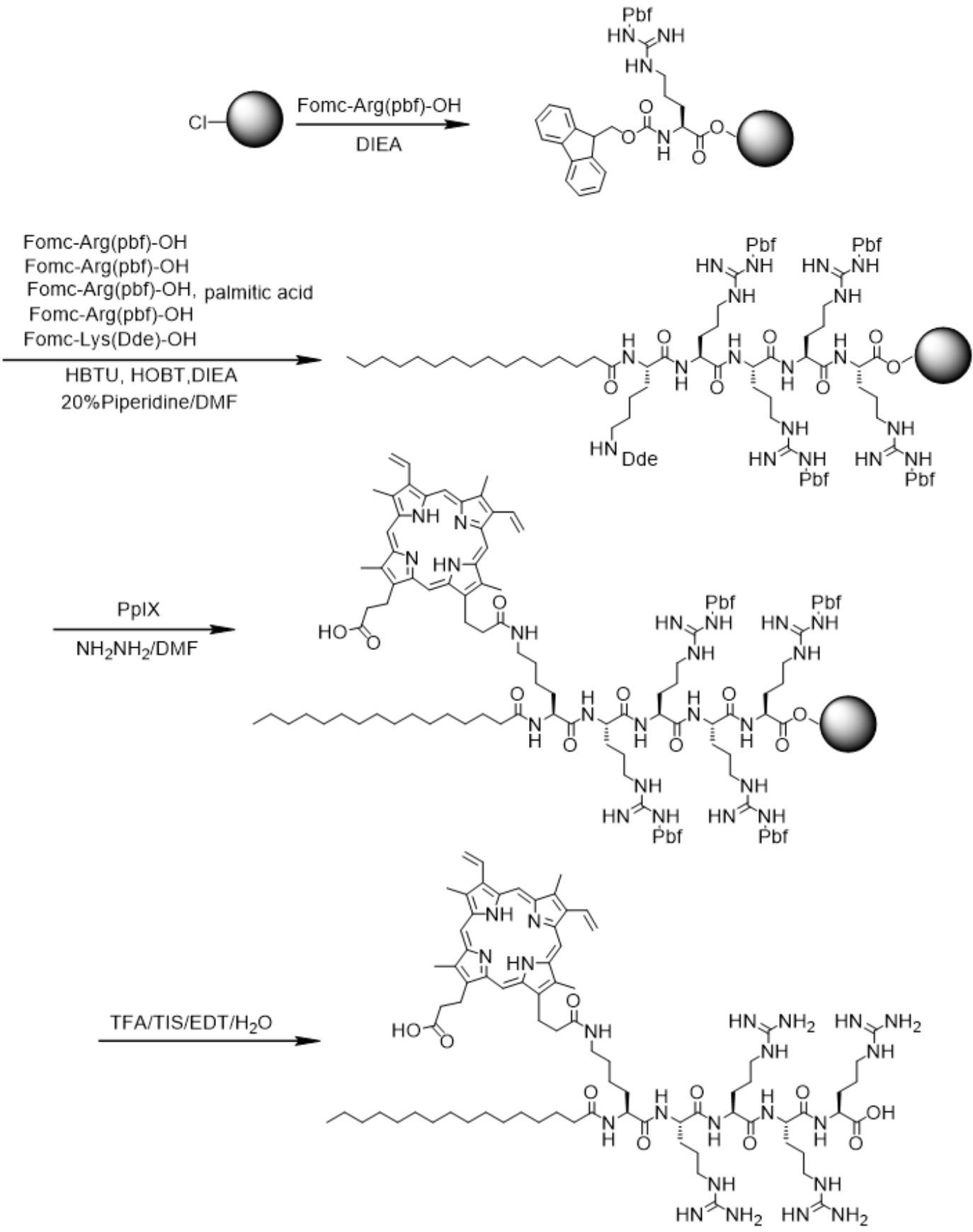

Figure S1. The synthetic route and molecular structure of $\mathrm{C}_{16}-\mathrm{PpIX}$. 


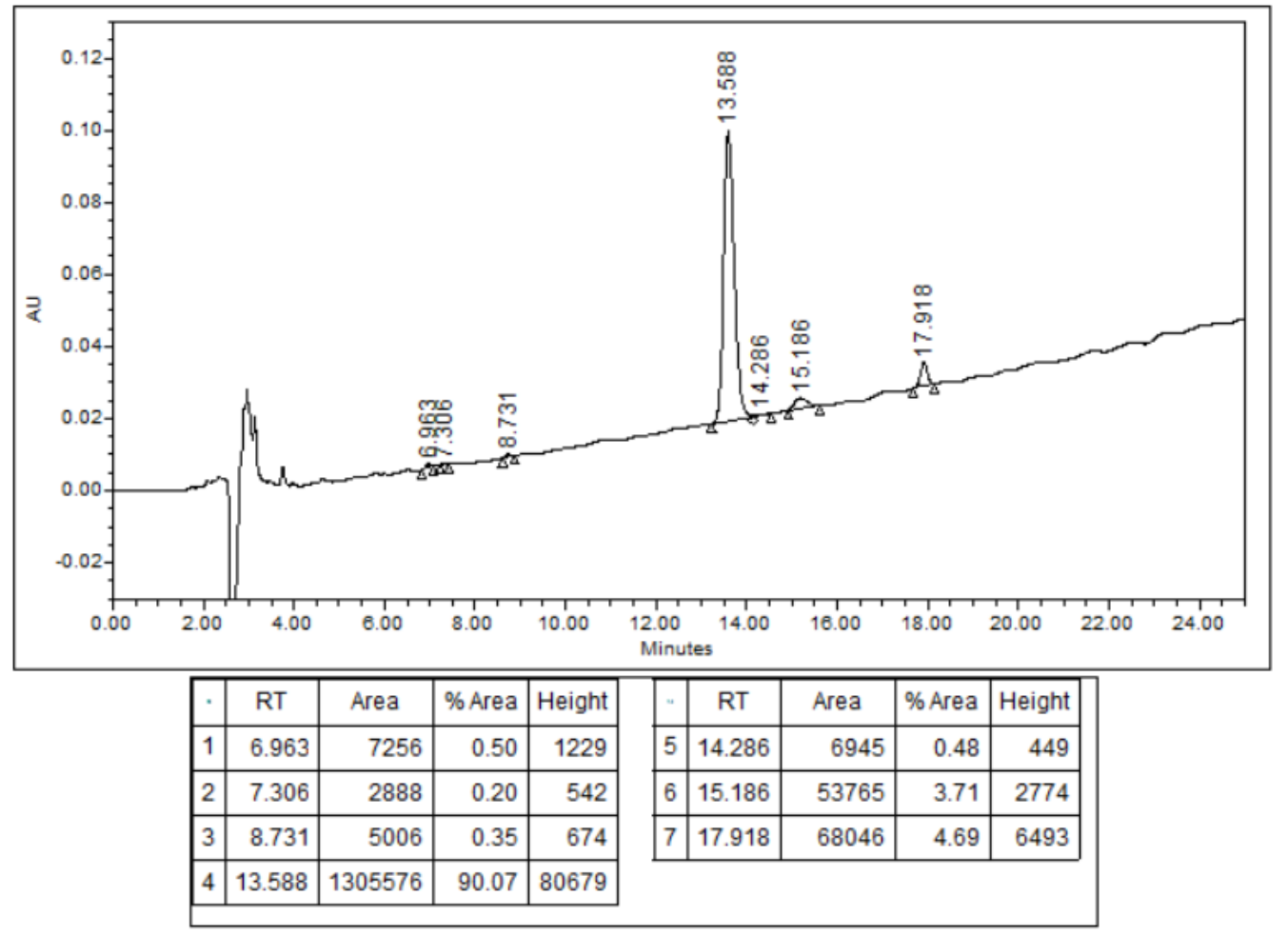

Figure S2. HPLC analysis of $\mathrm{C}_{16}-\mathrm{PpIX}$. 


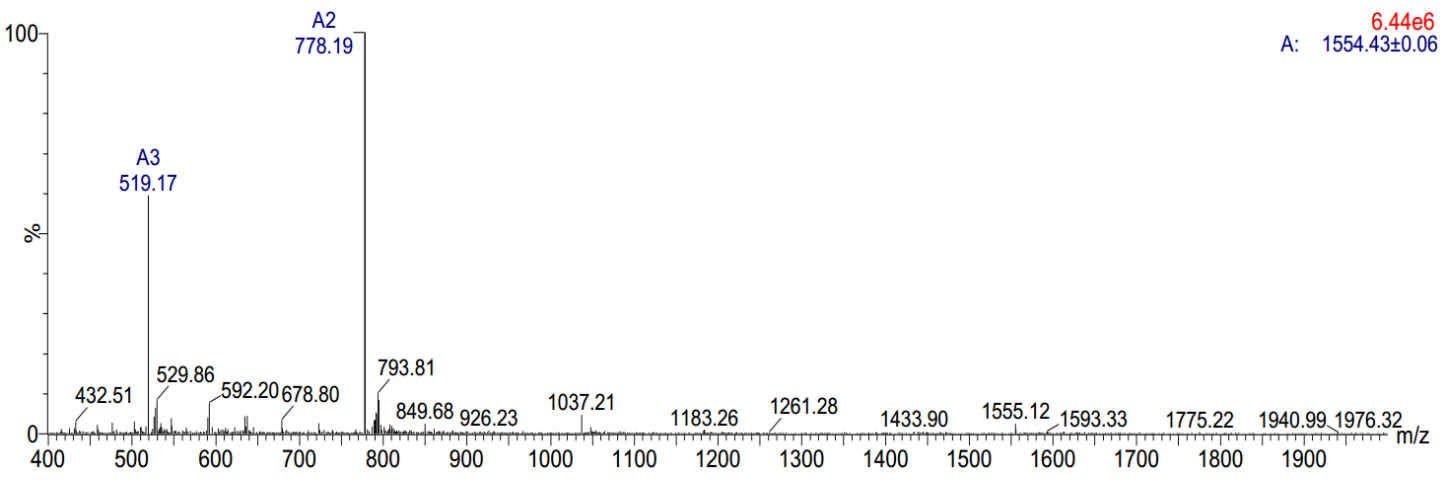

Figure S3. ESI-MS analysis of $\mathrm{C}_{16}$-PpIX.

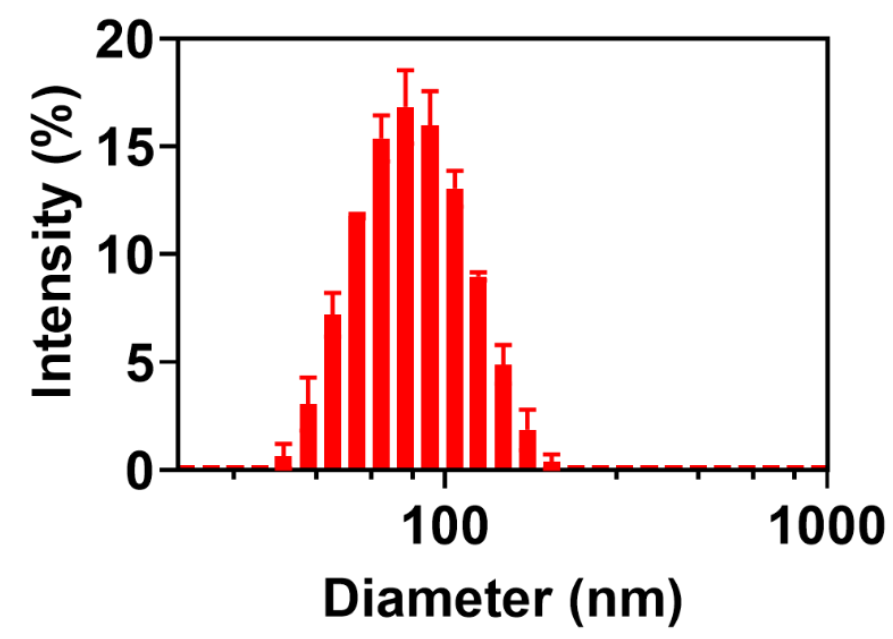

Figure S4. DSL analysis of copper peroxide. 


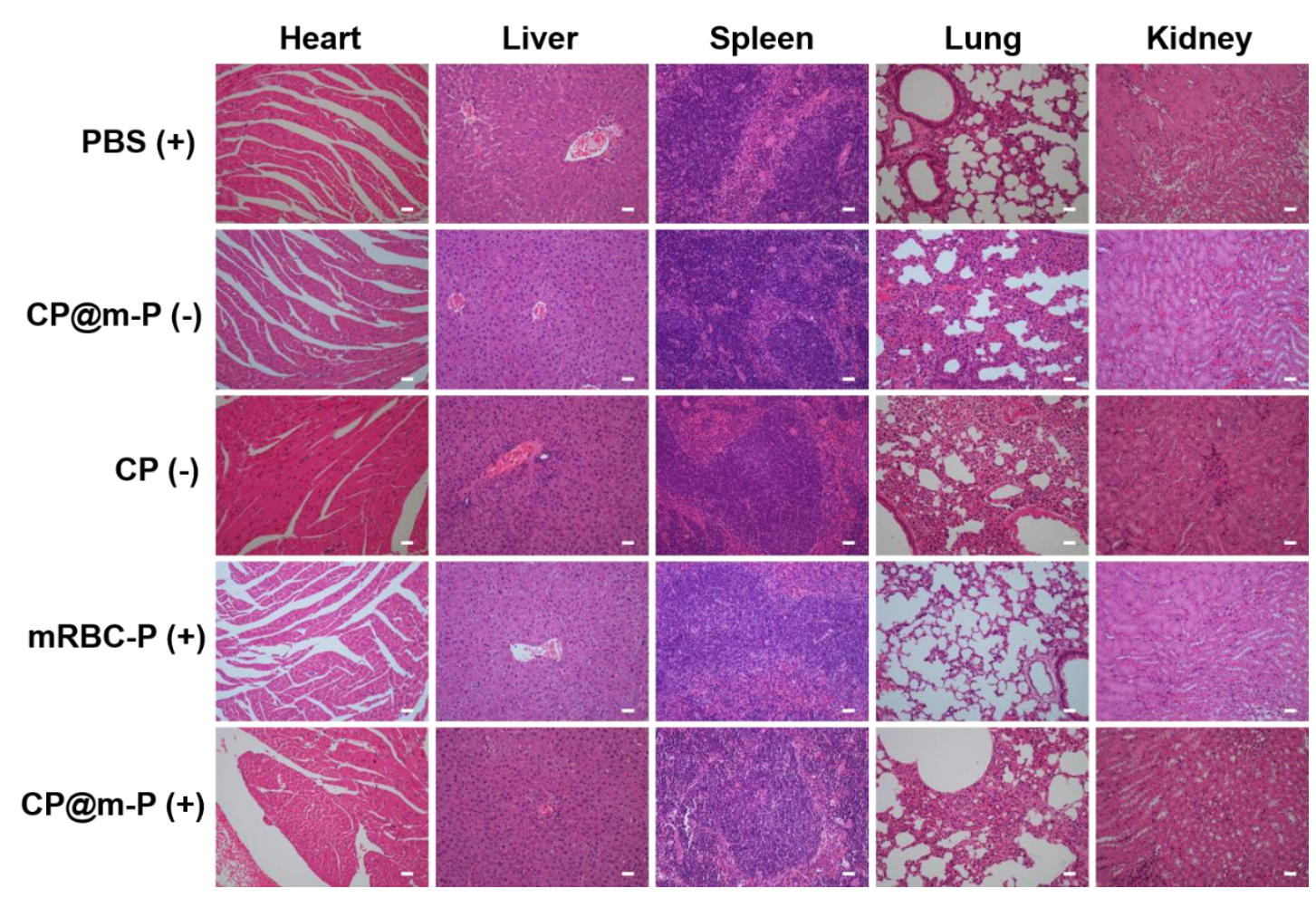

Figure S5. H\&E staining of main organs (heart, liver, spleen, lung, kidney) after 13day treatment. 


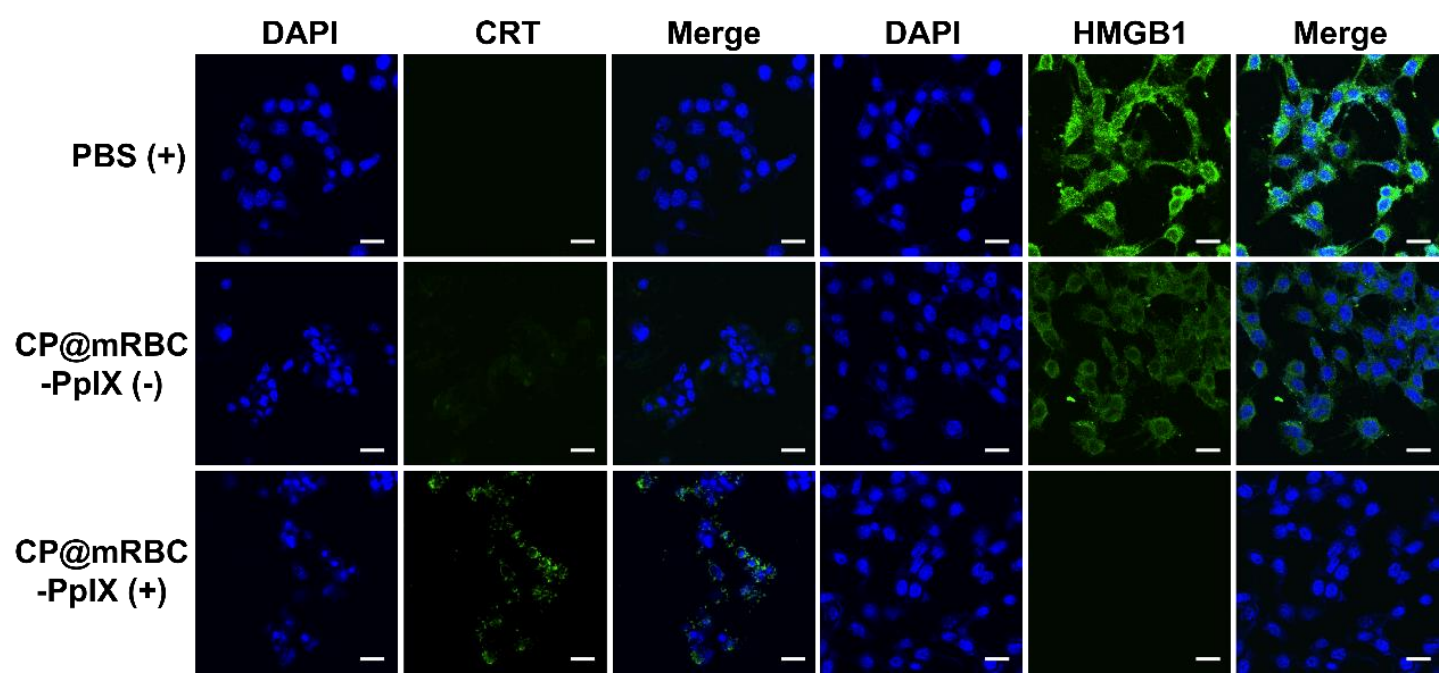

Figure S6. Immunofluorescence observation of CRT exposure on and HMGB1 release in the $4 \mathrm{~T} 1$ cells.

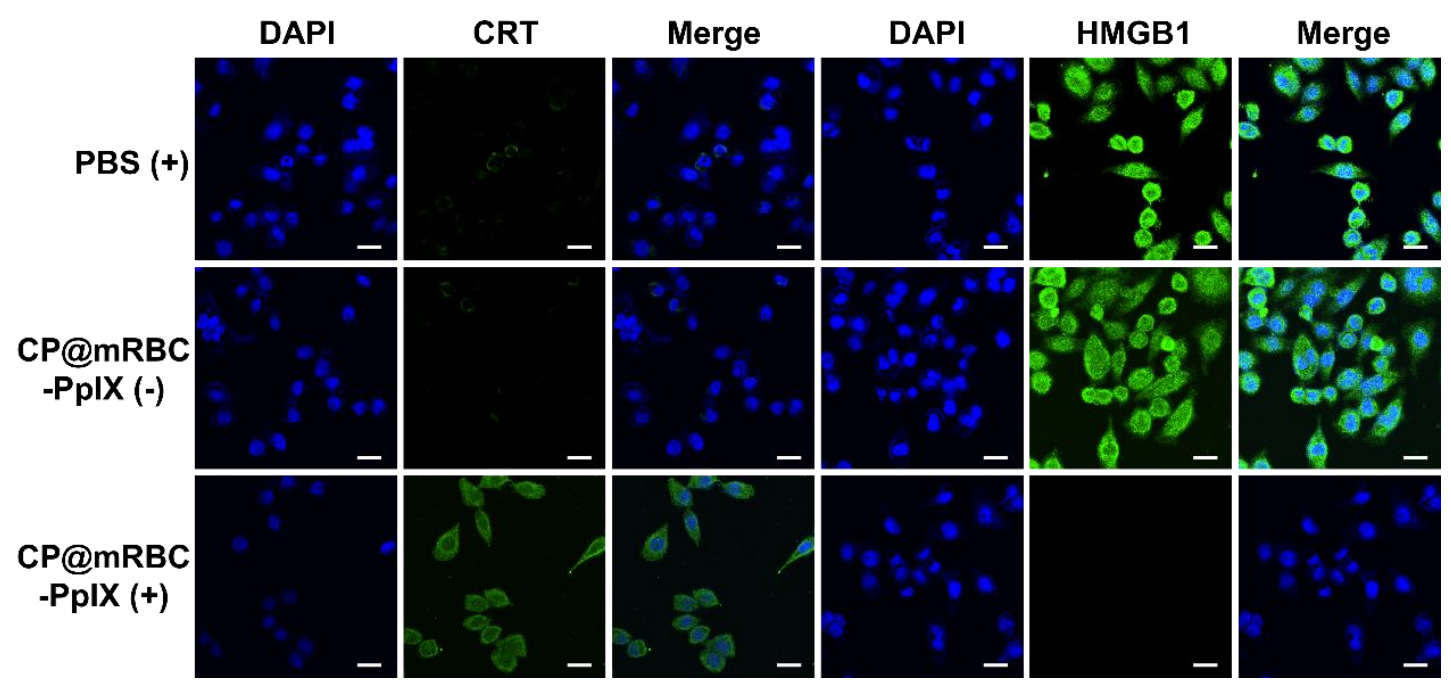

Figure S7. Immunofluorescence observation of CRT exposure on and HMGB1 release in the MCF-7 cells. 


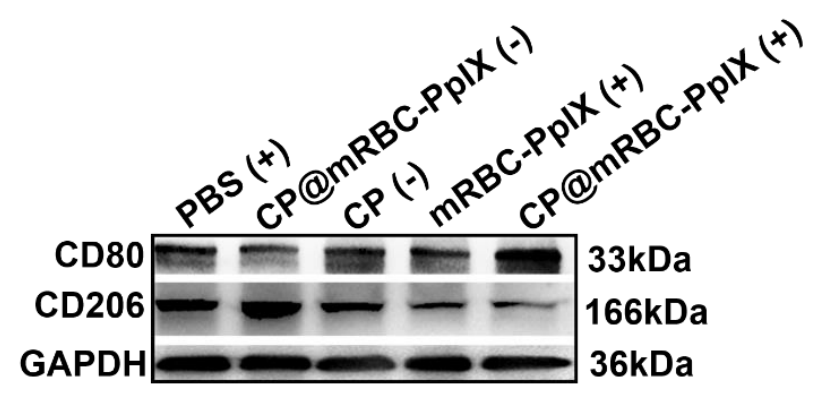

Figure S8. Western blotting of M1 and M2 population after different treatments. 